\title{
APPLICATION OF A DUAL-ENERGY MONOCHROMATIC X- RAY CT ALGORITHM TO POLYCHROMATIC X-RAY CT: A FEASIBILITY STUDY
}

\author{
S. CHANG ${ }^{1}$, H. K. $\mathrm{LEE}^{2}$, and G. $\mathrm{CHO}^{1 *}$ \\ ${ }^{1}$ Department of Nuclear Quantum Engineering, Korea Advanced Institute of Science and Technology \\ 335 Gwahak-ro, Yuseong-gu, Daejeon 305-701, Republic of Korea \\ ${ }^{2}$ Department of Mining and Nuclear Engineering, Missouri University of Science and Technology, Rolla \\ MO 65409, USA \\ *Corresponding author. E-mail : gscho@kaist.ac.kr
}

Received August 23, 2010

Accepted for Publication June 06, 2011

In this study, a simple post-reconstruction dual-energy computed tomography (CT) method is proposed. A dual-energy CT algorithm for monochromatic X-rays was adopted and applied to the dual-energy CT of polychromatic X-rays by assigning a representative mono-energy. The accuracy of algorithm implementation was tested with mathematical phantoms. To test the sensitivity of this algorithm to the inaccuracy of representative energy value in energy values, a simulation study was performed with mathematical phantom. To represent a polychromatic x-ray energy spectrum with a single-energy, mean energy and equivalent energy were used, and the results were compared. The feasibility of the proposed method was experimentally tested with two different micro-CTs and a test phantom made of polymethyl methacrylate (PMMA), water, and graphite. The dual-energy calculations were carried out with CT images of all possible energy pairs among 40, 50, 60, 70, and 80 kVp. The effective atomic number and the electron density values obtained from the proposed method were compared with theoretical values. The results showed that, except the errors in the effective atomic number of graphite, most of the errors were less than $10 \%$ for both CT scanners, and for the combination of $60 \mathrm{kVp}$ and $70 \mathrm{kVp}$, errors less than $6.0 \%$ could be achieved with a Polaris $90 \mathrm{CT}$. The proposed method shows simplicity of calibration, demonstrating its practicality and feasibility for use with a general polychromatic CT.

KEYWORDS : Computed Tomography(CT), Dual Energy, Micro CT

\section{INTRODUCTION}

Computed tomography (CT) has been indispensable in diagnostics since its advent in the early 1970s. CT imaging shows the inner structure of a patient or an object by visualizing the linear attenuation coefficient of each different component. Dual-energy CT (DECT), proposed in 1970s [1], can provide more information about the materials that comprise the scanned object than a conventional CT does. Many studies have been carried out on DECT imaging, and the first commercial clinical dual-source (or energy) CT was introduced in $2005^{1}$. It is equipped with two X-ray tube and detector pairs, and it simultaneously acquires projection data at two different

${ }^{1}$ http://www.medical.siemens.com energies. DECT images can be used for various purposes such as material differentiation, contrast enhancement of conventional CT [2], beam hardening correction [3, 4], attenuation correction for single photon emission computed tomography (SPECT) or positron emission tomography (PET) [5, 6], explosive detection [7], and luggage screening [8]. In addition, the electron density (ED) of scanned materials can be obtained with DECT. Accurate ED information of a patient's body is vital for precise treatment planning in the process of radiotherapy.

Techniques for extracting material information from DECT can be categorized into three groups: prereconstruction methods, post-reconstruction methods, and iterative reconstruction methods. Pre-reconstruction techniques [1, 7, 9-11] decompose the linear attenuation coefficient (LAC) into a photoelectric part and a Compton scattering part, or replace it with an equivalent LAC of known basis materials using basis material decomposition. These techniques can theoretically reconstruct images that 
are free of beam hardening artifacts but need careful and expensive calibration to reduce errors. Post-reconstruction techniques [12-16] extract material information from reconstructed $\mathrm{CT}$ images. These methods have an advantage in their computational simplicity because calculations are performed only at corresponding pixels of the CT images at two different energies. These are useful when raw projected data are not available or the detailed parameters of the reconstruction software are not adjustable. Both of these techniques use filtered back projection (FBP) for reconstruction, which does not consider the statistical nature of the radiation. Iterative DECT reconstruction techniques [17-19] might provide more accurate information, where CT images are reconstructed iteratively using a statistical model. These methods show good image quality but incur huge computational cost.

In addition to the categories mentioned above, methods using dual energies of monochromatic radiation have been proposed. A synchrotron [20, 21] or Ir-192 [22] isotope was used as the monochromatic radiation source of the DECT. These methods show high accuracy in extracting material properties, but their practicality is very low due to limited availability of the radiation sources.

We propose a simple and convenient DECT technique which could be used easily with a conventional x-ray CT. We focused on the simplicity of the post-reconstruction approach and the accuracy of the monochromatic radiation approach. We combined ideas from these two approaches, applying the DECT technique for monochromatic sources to DECT images obtained with polychromatic x-rays. We assigned a representative energy for each of the two different polychromatic spectra, with which we performed the DECT method originally developed for monochromatic sources. However, as we are adopting a monochromatic $\mathrm{x}$-ray technique with polychromatic $\mathrm{x}$-rays, the accuracy of DECT images may not be guaranteed anymore. In this study, we experimentally test the feasibility of this new approach and investigate the effect of the representative energy of a spectrum on the accuracy of results. The accuracy of the proposed method is examined by comparing the extracted values of the effective atomic number $Z_{\text {eff }}$ and the ED of the test phantom materials with theoretical values.

\section{MATERIALS AND METHODS}

\subsection{Overview of Dua- energy Methods}

Projection function $p$ at a given detector position in the simplest image formation model is calculated as

$$
p=-\ln \int S(E) \exp \left[-\int \mu(\vec{r}, E) d l\right] d E,
$$

where $S(E)$ is the X-ray energy spectrum, and $\mu(\vec{r}, E)$ is an energy dependent LAC at position $\vec{r}$. The LAC $\mu(E)$ can be expressed as a linear combination of basis functions as

$$
\mu(E)=a_{1} f_{1}(E)+a_{2} f_{2}(E)+a_{3} f_{3}(E)+\cdots+a_{n} f_{n}(E) .
$$

All the dual-energy methods solve Eqs. (1) and (2) with data sets acquired at two different energies using their own model of the LAC.

Alvarez and Macovski modeled the LAC as a linear combination of energy-dependent photoelectric and Compton interaction contribution as [1]

$$
\mu(E)=a_{1} f_{P E}(E)+a_{2} f_{K N}(E),
$$

where $f_{P E}(E)$ is the energy dependence of the photoelectric interaction, and $f_{K N}(E)$ is the Klein-Nishina formula.

Basis material methods set the LAC as a linear combination of LACs of two known materials [9]:

$$
\mu(E)=b_{1} \mu_{1}(E)+b_{2} \mu_{2}(E),
$$

where $b_{1}$ and $b_{2}$ are coefficients of physical properties of chosen materials, and $\mu_{1}(E)$ and $\mu_{2}(E)$ are mass attenuation coefficients of previously chosen materials at a given energy.

Neglecting the $Z$ dependence of the scattering cross section, Vinegar and Wellington approximated the LAC as [23]

$$
\mu(E)=\rho_{e}\left(Z_{e f f}^{n} P(E)+C(E)\right),
$$

where $P(E)$ and $C(E)$ are energy-only-dependent parts of the photoelectric and the scattering cross sections, respectively, and $\rho_{\mathrm{e}}$ is the ED. The exponent $n$ ranges from 3.0 to 4.0. After $P(E)$ and $C(E)$ are obtained from calibration with a known material, $Z^{\mathrm{n}}$ and $\rho_{\mathrm{e}}$ can be calculated from Eq. (5) when the LACs for low and high energies, $\mu\left(E_{L}\right)$ and $\mu\left(E_{H}\right)$, are provided.

\subsection{Proposed DECT Method}

In our study, the DECT algorithm developed for monochromatic x-rays [20, 21] was adopted because of its high accuracy and computational simplicity. This algorithm models the LAC as

$$
\mu(E)=\rho_{e}\left(Z^{4} \sigma_{P E}(Z, E)+\sigma_{S}(Z, E)\right),
$$

where $Z$ is the $Z_{e f f}$, and $\sigma_{P E}(Z, E)$ and $\sigma_{S}(Z, E)$ are the functions for the photo-electric absorption cross-section and for the scattering cross-section, respectively. Note that these functions depend not only on energy but also on $Z_{\text {eff, }}$ which differs from (5).

The ED is defined as

$$
\rho_{e}=\rho \frac{Z_{e f f}}{A} N_{A}
$$


where $\rho, A$ and $N_{A}$ are mass density, mass number, and Avogadro's number, respectively. The pixel value of a CT image obtained with monochromatic radiation can be treated as the LAC at that position of an object. From two reconstructed images at different energies, the LACs of the material are obtained, but $Z_{\text {eff }}$ and ED are still unknown as in following equations:

$$
\begin{aligned}
& \mu_{L}=\rho_{e}\left(Z^{4} \sigma_{P E}\left(Z, E_{L}\right)+\sigma_{S}\left(Z, E_{L}\right)\right) \\
& \mu_{H}=\rho_{e}\left(Z^{4} \sigma_{P E}\left(Z, E_{H}\right)+\sigma_{S}\left(Z, E_{H}\right)\right),
\end{aligned}
$$

where $\mu_{L}$ and $\mu_{H}$ are the LACs at low and high energies, respectively, and are the pixel values of reconstructed images as well. To determine the initial photo-electric absorption and scattering cross-section Z-independent approximation is performed.

$$
\begin{gathered}
Z^{(0)}=\left[\frac{\mu_{H} \sigma_{S}\left(E_{L}\right)-\mu_{L} \sigma_{S}\left(E_{H}\right)}{\mu_{L} \sigma_{P E}\left(E_{H}\right)-\mu_{H} \sigma_{P E}\left(E_{L}\right)}\right]^{1 / 4}, \\
\rho_{e}^{(0)}=\frac{\mu_{L} \sigma_{P E}\left(E_{H}\right)-\mu_{H} \sigma_{P E}\left(E_{H}\right)}{\sigma_{P E}\left(E_{H}\right) \sigma_{S}\left(E_{L}\right)-\sigma_{P E}\left(E_{L}\right) \sigma_{S}\left(E_{H}\right)} .
\end{gathered}
$$

Then, problems are solved iteratively until the values of $Z_{\text {eff }}$ and ED converge:

$$
Z^{(i+1)}=\left[\frac{\mu_{H} \sigma_{S}\left(E_{L}, Z^{(i)}\right)-\mu_{L} \sigma_{S}\left(E_{H}, Z^{(i)}\right)}{\mu_{L} \sigma_{P E}\left(E_{H}, Z^{(i)}\right)-\mu_{H} \sigma_{P E}\left(E_{L}, Z^{(i)}\right)}\right]^{1 / 4},
$$

$\rho_{e}^{(i+1)}=\frac{\mu_{L} \sigma_{P E}\left(E_{H}, Z^{(i+1)}\right)-\mu_{H} \sigma_{P E}\left(E_{L}, Z^{(i+1)}\right)}{\sigma_{P E}\left(E_{H}, Z^{(i+1)}\right) \sigma_{S}\left(E_{L}, Z^{(i+1)}\right)-\sigma_{P E}\left(E_{L}, Z^{(i+1)}\right) \sigma_{S}\left(E_{H}, Z^{(i+1)}\right)}$.

To evaluate the accuracy and computational simplicity of the algorithm, it was implemented in $\mathrm{C}$ language and tested with a mathematical phantom consisting of graphite, water, and polymethyl methacrylate (PMMA). Theoretical values [24] used for the properties of the phantom materials are summarized in Table 1. Monochromatic X-rays peaked at $40 \mathrm{keV}, 50 \mathrm{keV}, 60 \mathrm{keV}, 70 \mathrm{keV}$, or $80 \mathrm{keV}$ were used in the test. The size of the CT images was 512 x 512, and it took 1.9 seconds, utilizing the OpenMP ${ }^{2}$, to produce images of $Z_{e f f}$ and ED of the mathematical phantom on an AMD quad-core Phenom $98502.5 \mathrm{GHz}$ CPU. After six iterations, the values converged. Iteration stopped when all of the difference between the current step and the immediately prior step appeared at the 3rd digit below the decimal point.

Images of $Z_{\text {eff }}$ and ED of the mathematical phantom calculated from two (40 keV and $80 \mathrm{keV}$ ) monochromatic

\begin{tabular}{|c|c|c|c|}
\hline Property & Graphite & Water & PMMA \\
\hline Density $\left(\mathrm{g} / \mathrm{cm}^{3}\right)$ & 1.79 & 1.0 & 1.17 \\
\hline Electron density $\left(\mathrm{x} 10^{23}\right.$ electrons $\left./ \mathrm{cm}^{3}\right)$ & 5.39 & 3.34 & 3.84 \\
\hline Effective atomic number & 6.0 & 7.51 & 6.56 \\
\hline
\end{tabular}
$\mathrm{X}$-ray CT images are shown in Figure 1, and the percentage errors of the calculated values of $Z_{\text {eff }}$ and ED for various

${ }^{2} \mathrm{http}: / /$ www.openmp.org

Table 1. Material Properties of the Mathematical Phantom used in the Test

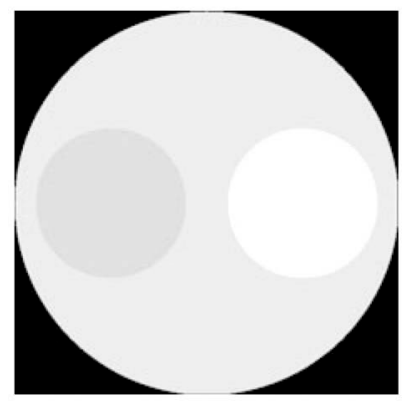

(a)

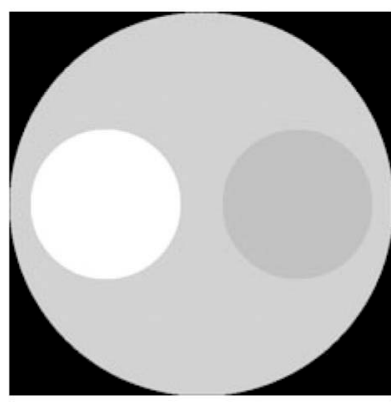

(b)

Fig. 1. Effective Atomic Number Image (a) and Electron Density Image (b) of a Mathematical Phantom which Consists of Graphite, water, and PMMA. CT Images Obtained with $40 \mathrm{keV}$ and $80 \mathrm{keV}$ Monochromatic x-rays were used to Calculate $Z_{\text {eff }}$ and ED. 
combinations of low and high energies are listed in Table 2. For all combinations of the energies, the errors in ED are less than $1.0 \%$, and with the combination of $60 \mathrm{keV}$ and $70 \mathrm{keV}$, the errors in $Z_{\text {eff }}$ are less than $1 \%$ as well, verifying the high accuracy of the method for dual energies of monochromatic x-rays.

To successfully apply the DECT algorithm to polychromatic $x$-rays, it must not be sensitive to the inaccuracy of representative energy values. Using the same mathematical phantom, this behavior was investigated for the case of the $40 \mathrm{keV}$ and $80 \mathrm{keV}$ combination. The errors in the resulting $Z_{\text {eff }}$ and ED when the errors were intentionally added to the low and high energy values are shown in Figure 2 for the case of graphite. When up to $\pm 20 \%$ of errors are added to the energies, the errors in $Z_{\text {eff }}$ range from $-50 \%$ to $40 \%$, while those in ED range from $10 \%$ to $10 \%$, which is half of the error range introduced in energies. The results show that the DECT method for calculation of the ED is not too sensitive to uncertainties in energy values. This method, therefore, may be applied to polychromatic $\mathrm{x}$-rays for extraction of electron density information if a proper energy value is assigned to represent the spectrum. This method may not be suitable for extraction of the effective atomic number information from DECT of polychromatic x-rays due to large errors.

Table 2. Percentage Errors in the Values of $Z_{\text {eff }}$ and ED of the Mathematical Phantom Materials Calculated from Dual-energy Monochromatic x-ray CT Images.

\begin{tabular}{|c|c|c|c|c|c|c|c|}
\hline \multirow[b]{2}{*}{$\mathrm{E}_{\mathrm{L}}$} & \multirow[b]{2}{*}{$\mathrm{E}_{\mathrm{H}}$} & \multicolumn{3}{|c|}{$Z_{\text {eff }}$ errors ${ }^{*}$} & \multicolumn{3}{|c|}{ Electron Density errors* } \\
\hline & & \multirow{2}{*}{ Graphite } & \multirow{2}{*}{ Water } & \multirow{2}{*}{ PMMA } & \multirow{2}{*}{ Graphite } & \multirow{2}{*}{ Water } & \multirow{2}{*}{ PMMA } \\
\hline \multicolumn{2}{|c|}{$(\mathrm{keV})$} & & & & & & \\
\hline \multirow[t]{4}{*}{40} & 50 & -1.89 & -1.85 & -1.88 & -0.12 & -0.42 & 0.36 \\
\hline & 60 & -1.82 & -2.02 & -1.88 & -0.16 & -0.26 & 0.36 \\
\hline & 70 & -1.65 & -1.80 & -1.52 & -0.24 & -0.46 & 0.12 \\
\hline & 80 & -1.90 & -2.14 & -1.66 & -0.12 & -0.15 & 0.21 \\
\hline \multirow[t]{3}{*}{50} & 60 & -1.66 & -2.33 & -1.89 & -0.19 & -0.15 & 0.36 \\
\hline & 70 & -1.35 & -1.74 & -1.08 & -0.28 & -0.48 & 0.05 \\
\hline & 80 & -1.90 & -2.42 & -1.44 & -0.12 & -0.10 & 0.19 \\
\hline \multirow[t]{2}{*}{60} & 70 & -0.79 & -0.74 & 0.33 & -0.36 & -0.73 & -0.20 \\
\hline & 80 & -2.18 & -2.53 & -0.95 & -0.09 & -0.08 & 0.13 \\
\hline 70 & 80 & -4.57 & -5.60 & -3.15 & 0.13 & 0.45 & 0.39 \\
\hline
\end{tabular}

${ }^{*}$ Errors were calculated based on the theoretical values in Table 1.

Error $=100 \mathrm{x}($ calculated value - theoretical value $) /$ theoretical value

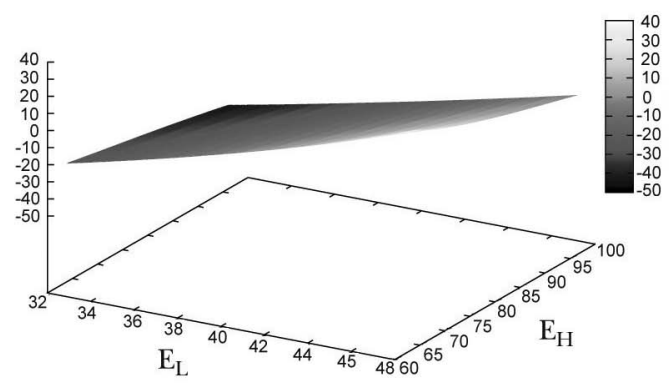

(a)

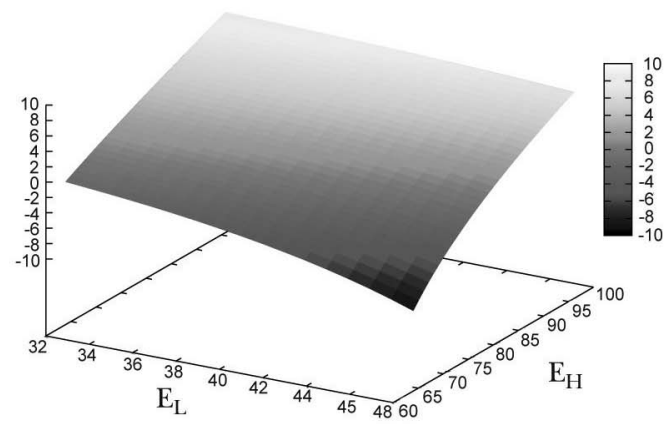

(b)

Fig. 2. Errors in $Z_{\text {eff }}$ (a) and ED (b) of the Water in the Mathematical Phantom when Up to $\pm 20 \%$ of Errors are Added to the Low and High Energy Values. 


\subsection{Application to Polychromatic x-rays}

The feasibility tests of the DECT method to polychromatic $\mathrm{X}$-rays were carried out through phantom imaging experiments with two micro-CT scanners: Nano Focus Ray (NFR) Polaris G90 and DRGEM Harmony 90. The x-ray source of the Polaris G90 was a Kevex PXS5-927 tube, and its voltage could be adjusted from $20 \mathrm{kVp}$ to $90 \mathrm{kVp}$ with the maximum power of $8 \mathrm{~W}$. The image detector was a Hamamatsu C7942CA-22 image sensor with $2240 \times 2344$ active pixels and a $50 \mu \mathrm{m}$ pixel pitch. It had a slip ring structure for fast rotation and 360 radiographic images were acquired in a revolution for 5 minutes. Harmony 90 was equipped with the same x-ray tube as the Polaris G90. For the image detector, a RadIcon Shad-o-Box 2048 X-ray camera with $2048 \times 1024$ pixels was used. It used a motor-driven rotation mechanism, and 400 views were acquired in a revolution for 20 minutes. Both scanners used the FDK algorithm for image reconstruction and the image size was $512 \times 512$ pixels.

CT images of a test phantom were acquired at five different energies of $40 \mathrm{kVp}, 50 \mathrm{kVp}, 60 \mathrm{kVp}, 7 \mathrm{kVp}$, and $80 \mathrm{kVp}$. Filtration of $\mathrm{x}$-rays provides a narrower spectrum and lessens the hardening effect, and a $0.5 \mathrm{~mm}$ aluminum filter was used in both scanners with an X-ray tube current of $50 \mu \mathrm{A}$.

The mathematical phantom described in Section 2.2 was fabricated for real experiments. Water, graphite and PMMA were used as the phantom materials as shown in Figure 3(a). In a PMMA cylinder of $30 \mathrm{~mm}$ diameter, a graphite cylinder of $8 \mathrm{~mm}$ diameter was inserted. It is shown as the dark area in Figure 3(a). A hole of $10 \mathrm{~mm}$ diameter in the PMMA cylinder was filled with distilled water. It is shown as the gray area in Figure 3(a). The measured densities of graphite and PMMA were 1.79 $\mathrm{g} / \mathrm{cm}^{3}$ and $1.19 \mathrm{~g} / \mathrm{cm}^{3}$, respectively.

To use the iterative process of Eqs. (9) to (12) to get the information of $Z_{\text {eff }}$ and ED, two specific energies $\left(E_{L}\right.$ and $E_{H}$ ) which represent the high and low x-ray energy spectra must be determined. In this study, we adopted two representative energies: mean energy and equivalent energy. The mean energy was obtained by simple calculation of the mean value of the spectrum. The equivalent energy was obtained by finding a monochromatic energy which has the same half-value layer (HVL) as the polychromatic energy spectrum. The HVL of the polychromatic energy spectrum was obtained by seeking a value of $\mathrm{x}$ which satisfied the following condition:

$$
I(x) \sum_{i=1}^{N} I_{o i} \exp \left(-\mu_{i} x\right)=\frac{1}{2} \sum_{i=1}^{N} I_{o i}
$$

where the polychromatic energy spectrum has been discretized into $N$ energy bins, and $I_{o i}$ and $\mu_{i}$ indicate the intensity and attenuation coefficient of i-th energy bin, respectively.

The energy spectra from the micro CTs used in the experiment were simulated using SpekCalc [25]. The simulated $\mathrm{x}$-ray energy spectra, which were normalized to have the same intensity, are shown in Figure 4, and the corresponding mean energies and equivalent energies are listed in Table 3. Combinations of these energies were used as the low and high energies in Eqs. (9)-(12) to obtain $Z_{\text {eff }}$ and $\mathrm{ED}$ values, and the results were compared.

The CT numbers of the micro-CTs were not calibrated for all energies used in the experiment. To convert reconstructed CT numbers to the physical LACs, the following equation was used.

$$
\mu(E)=\left(\frac{H-H_{\text {air }}}{H_{\text {water }}-H_{\text {air }}}\right) \cdot \mu_{\text {water }}(E) \text {, }
$$

where $H$ indicates a $\mathrm{CT}$ number at the corresponding position. The LACs of water were obtained from the National Institute of Standards and Technology (NIST; http://www.nist.gov/physlab/data/xcom/ index.cfm), and cubic-spline interpolation was used for energies not in the LAC table.

\section{RESULTS}

CT images of the phantom were obtained by two different scanners with the same operation conditions, namely, tube voltage, beam current, detector exposure time, and filter. Profiles of CT images taken at $40 \mathrm{keV}$ with the two scanners are shown in Figure 3(b). The CT numbers are different between the two scanners because these were not calibrated at this energy.

Table 3. Mean Energies and Equivalent Energies of the x-ray Spectra used in the Experiment.

\begin{tabular}{c|c|c|c|c|c}
\hline & \multicolumn{5}{|c}{ Peak Energy $(\mathrm{keV})$} \\
\cline { 2 - 6 } & 40 & 50 & 60 & 70 & 22.3 \\
\hline Equi $^{*}$ & 18.1 & 19.7 & 21.0 & 34.74 & 38.6 \\
\hline Emean $^{*}$ & 23.86 & 27.68 & 31.28 & 32.32 \\
\hline
\end{tabular}

"Calculated from x-ray spectra simulated with SpekCalc. 


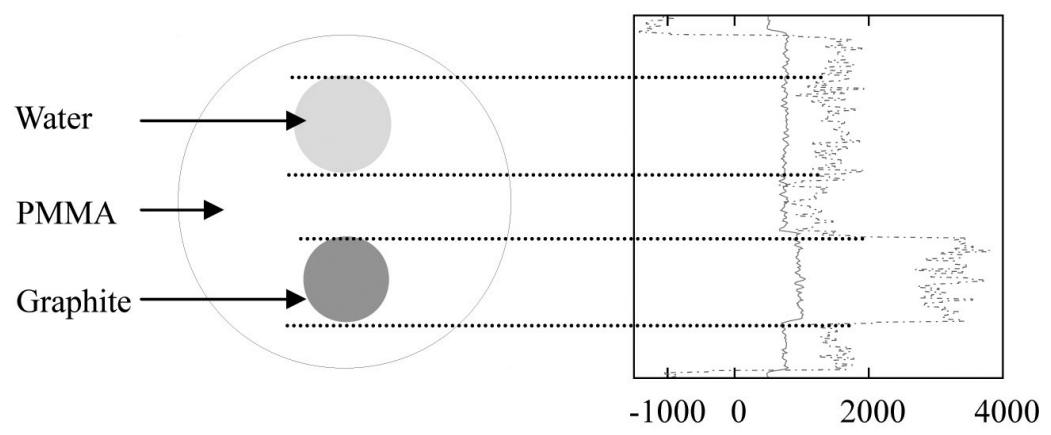

(a)

(b)

Fig. 3. Cross-sectional Image of the Phantom (a) and Profiles (b) of CT Images from Two Different Scanners. The Phantom Consists of PMMA, Water, and Graphite. Solid and Dashed Lines Indicate Profiles of the Phantom Image Obtained with Harmony 90 and Polaris G90, Respectively.

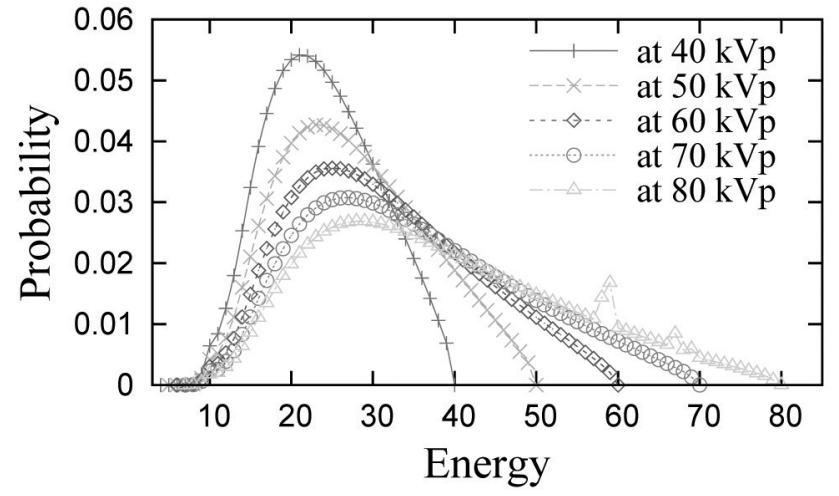

Fig. 4. Simulated x-ray Energy Spectra of the CT Normalized to have the Same Intensity.

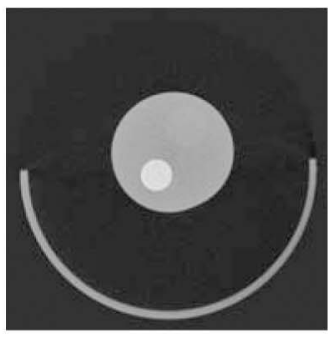

(a)

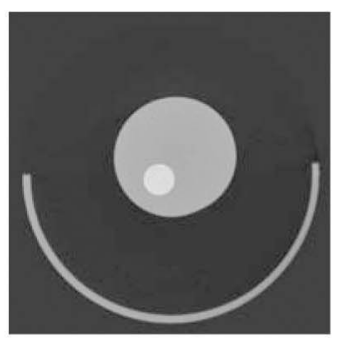

(b)

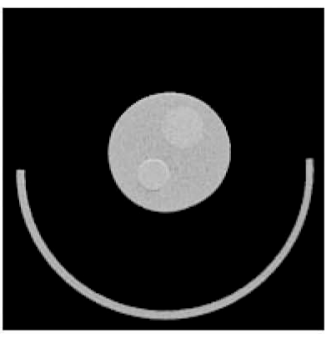

(c)

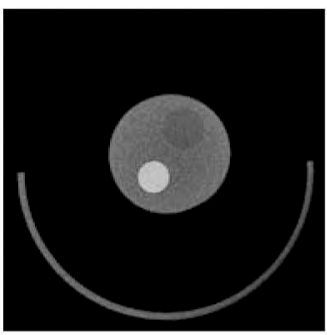

(d)

Fig. 5. CT Images of the Phantom Taken with Polaris G90 Scanner. CT Image at $40 \mathrm{kVp}$ (a), CT Image at $80 \mathrm{kVp}$ (b), $Z_{\text {eff }}$ Image (c), and ED Image (d). Mean Energies are used to Represent the Polychromatic Spectra.

For each combination of low and high energies, $Z_{\text {eff }}$ and ED images were extracted from the CT images. Figure 5 shows $\mathrm{CT}$ images of the phantom taken with the Polaris G90 scanner at $40 \mathrm{kVp}$ and $80 \mathrm{kVp}$ along with the corresponding $Z_{\text {eff }}$ and ED images when the mean energies were used. While the water and the PMMA regions are barely or hardly distinguishable in the original CT images, they are more distinct in the $Z_{\text {eff }}$ and ED images.

The extracted values of $Z_{\text {eff }}$ and ED are compared with the theoretical values from Table 1, and the percentage 


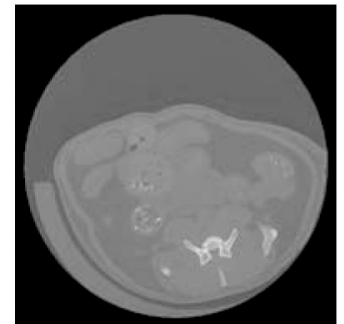

(a)

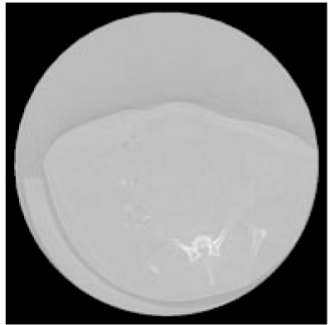

(b)

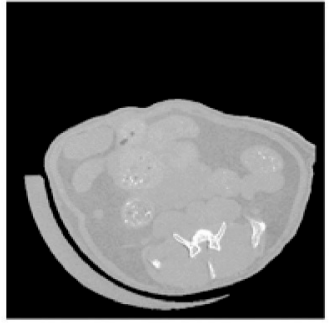

(c)

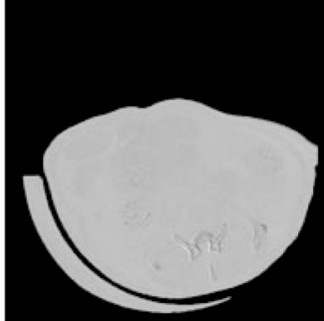

(d)

Fig. 6. CT Images of a Mouse Taken with Polaris G90. CT Image at $40 \mathrm{kVp}$ (a), CT Image at $80 \mathrm{kVp}$ (b), $Z_{\text {eff }}$ Image (c), and ED Image (d).

Table 4. Percentage Errors in Extracted $Z_{\text {eff }}$ and ED of the Phantom Materials. Equivalent Energies were used in Calculations. CT Images were Taken with Polaris G90.

\begin{tabular}{|c|c|c|c|c|c|c|c|}
\hline \multirow[b]{2}{*}{$\mathrm{E}_{\mathrm{L}}$} & \multirow[b]{2}{*}{$\mathrm{E}_{\mathrm{H}}$} & \multicolumn{3}{|c|}{ Errors of $Z_{\text {eff }}$} & \multicolumn{3}{|c|}{ Errors of ED } \\
\hline & & Graphite & Water & PMMA & Graphite & Water & PMMA \\
\hline \multicolumn{2}{|c|}{$(\mathrm{kVp})$} & \multicolumn{6}{|c|}{$(\%)$} \\
\hline \multirow[t]{4}{*}{40} & 50 & 43.33 & 5.35 & 13.77 & 23.29 & 58.00 & 51.75 \\
\hline & 60 & 49.93 & 18.27 & 14.62 & -3.33 & 4.98 & 17.20 \\
\hline & 70 & 44.98 & 18.87 & 8.81 & -9.84 & -9.26 & 8.24 \\
\hline & 80 & 34.32 & 14.89 & 10.10 & -9.10 & -14.02 & -2.47 \\
\hline \multirow[t]{3}{*}{50} & 60 & 47.76 & 9.96 & 18.63 & 17.25 & 43.03 & 32.96 \\
\hline & 70 & 47.43 & 23.34 & 19.91 & -9.26 & -8.10 & 4.26 \\
\hline & 80 & 36.30 & 21.73 & 19.04 & -9.38 & -16.79 & -9.60 \\
\hline \multirow[t]{2}{*}{60} & 70 & 56.59 & 22.62 & 22.75 & 1.71 & 14.17 & 19.87 \\
\hline & 80 & 47.01 & 26.14 & 31.82 & -9.65 & -14.38 & -13.41 \\
\hline 70 & 80 & 51.58 & 21.37 & 36.90 & 6.56 & 10.50 & -2.65 \\
\hline
\end{tabular}

errors are listed in Tables 4 to 7 . Tables 4 and 5 list errors that occurred when equivalent energies were used for calculations, and Tables 6 and 7 show the results that occurred when mean energies were used.

From the results, it is clear that when the mean energy was used to represent a spectrum, better accuracy could be achieved. Tables 6 and 7 show that, except the errors in $Z_{\text {eff }}$ of graphite, most of the errors are less than $10 \%$ for both CT scanners, and for the combination of $60 \mathrm{kVp}$ and $70 \mathrm{kVp}$, errors less than $6.0 \%$ could be achieved with the Polaris 90.

Although the results are not as accurate as those obtained with monochromatic radiation, applying the DECT algorithm for monochromatic radiation to polychromatic radiation seemed feasible, particularly to extract electron density information.

\section{DISCUSSION}

While the equivalent energy is the traditional representation of a polychromatic X-ray spectrum which is widely used in radiological physics, it did not suit our purpose for this study. Instead, the mean energy, which is higher than the equivalent energy, produced better results in terms of accuracy. The dual energy images with the energy pair of $40 \mathrm{kVp}$ and $80 \mathrm{kVp}$ yielded the best result among all the energy combinations. Compared to water and PMMA, errors in $Z_{\text {eff }}$ of graphite were much higher for all combinations of energies with both scanners. This DECT method may be limited to certain materials, and further studies with more materials are required for assessment of the material dependence.

The proposed method can be used to simply obtain $Z_{\text {eff }}$ 
Table 5. Percentage Errors in Extracted $Z_{\text {eff }}$ and ED of the Phantom Materials. Equivalent Energies were used in Calculations. CT Images were Taken with Harmony 90.

\begin{tabular}{|c|c|c|c|c|c|c|c|}
\hline \multirow[b]{2}{*}{$\mathrm{E}_{\mathrm{L}}$} & \multirow[b]{2}{*}{$\mathrm{E}_{\mathrm{H}}$} & \multicolumn{3}{|c|}{ Errors of $Z_{e f f}$} & \multicolumn{3}{|c|}{ Errors of ED } \\
\hline & & Graphite & Water & PMMA & Graphite & Water & PMMA \\
\hline \multicolumn{2}{|c|}{$(\mathrm{kVp})$} & \multicolumn{6}{|c|}{$(\%)$} \\
\hline \multirow[t]{4}{*}{40} & 50 & 36.00 & 3.20 & 18.29 & 43.30 & 66.89 & 42.89 \\
\hline & 60 & 54.67 & 16.91 & 23.48 & -9.15 & 6.11 & 6.54 \\
\hline & 70 & 40.67 & 21.30 & 19.66 & -4.23 & -7.87 & 0.39 \\
\hline & 80 & 46.67 & 15.71 & 16.92 & -20.95 & -13.26 & -9.51 \\
\hline \multirow[t]{3}{*}{50} & 60 & 51.17 & 9.19 & 14.48 & 18.81 & 46.59 & 50.81 \\
\hline & 70 & 44.83 & 26.36 & 19.97 & -0.87 & -4.49 & 9.79 \\
\hline & 80 & 60.17 & 23.30 & 20.88 & -25.77 & -15.48 & -10.08 \\
\hline \multirow[t]{2}{*}{60} & 70 & 35.67 & 15.05 & 21.34 & 29.52 & 27.51 & 38.54 \\
\hline & 80 & 64.83 & 25.03 & 31.25 & -19.72 & -13.14 & -9.17 \\
\hline 70 & 80 & 60.17 & 13.58 & 30.18 & 2.91 & 28.44 & 11.22 \\
\hline
\end{tabular}

Table 6. Percentage Errors in Extracted $Z_{\text {eff }}$ and ED of the Phantom Materials. Mean Energies were used in Calculations. CT Images were Taken with Polaris G90.

\begin{tabular}{|c|c|c|c|c|c|c|c|}
\hline \multirow[b]{2}{*}{$\mathrm{E}_{\mathrm{L}}$} & \multirow[b]{2}{*}{$\mathrm{E}_{\mathrm{H}}$} & \multicolumn{3}{|c|}{ Errors of $Z_{e f f}$} & \multicolumn{3}{|c|}{ Errors of ED } \\
\hline & & Graphite & Water & PMMA & Graphite & Water & PMMA \\
\hline \multicolumn{2}{|c|}{$(\mathrm{kVp})$} & \multicolumn{6}{|c|}{$(\%)$} \\
\hline \multirow[t]{4}{*}{40} & 50 & 29.29 & 10.77 & 13.02 & -0.28 & 0.71 & -5.46 \\
\hline & 60 & 17.79 & -5.72 & -0.11 & 9.72 & 7.92 & -0.22 \\
\hline & 70 & 18.29 & -5.52 & -0.06 & 8.12 & 5.99 & -1.18 \\
\hline & 80 & 19.23 & -4.18 & 2.81 & 6.16 & 2.66 & -6.24 \\
\hline \multirow[t]{3}{*}{50} & 60 & 11.70 & -6.62 & -0.42 & 18.03 & 14.71 & 4.33 \\
\hline & 70 & 15.10 & -7.52 & -2.27 & 11.29 & 8.36 & 0.60 \\
\hline & 80 & 17.46 & -4.87 & 2.93 & 7.61 & 3.32 & -6.34 \\
\hline \multirow[t]{2}{*}{60} & 70 & 21.35 & 0.59 & 5.98 & 5.83 & 3.12 & -2.70 \\
\hline & 80 & 22.09 & -0.67 & 8.00 & 3.78 & -0.84 & -10.25 \\
\hline 70 & 80 & 25.15 & 7.92 & 26.23 & 2.25 & -4.07 & -16.58 \\
\hline
\end{tabular}

and ED images from dual-energy CT images; however, this method is subject to artifacts on CT images because it uses the CT images directly. Therefore, artifact removal and noise reduction from the CT images before extraction of $Z_{\text {eff }}$ and ED images will enhance the quality of the results.
The proposed method was applied in DECT imaging of a small animal. CT images of a mouse at energies of $40 \mathrm{kVp}$ and $80 \mathrm{kVp}$ were acquired with a Polaris G90 scanner. Slices of abdomen were chosen, and the $Z_{\text {eff }}$ and ED images from the chosen CT images were calculated 
Table 7. Percentage Errors in Extracted $Z_{\text {eff }}$ and ED of the Phantom Materials. Mean Energies were used in Calculations. CT Images were Taken with Harmony 90.

\begin{tabular}{|c|c|c|c|c|c|c|c|}
\hline \multirow[b]{2}{*}{$\mathrm{E}_{\mathrm{L}}$} & \multirow[b]{2}{*}{$\mathrm{E}_{\mathrm{H}}$} & \multicolumn{3}{|c|}{ Errors of $Z_{\text {eff }}$} & \multicolumn{3}{|c|}{ Errors of ED } \\
\hline & & Graphite & Water & PMMA & Graphite & Water & PMMA \\
\hline \multicolumn{2}{|c|}{$(\mathrm{kVp})$} & \multicolumn{6}{|c|}{$(\%)$} \\
\hline \multirow[t]{4}{*}{40} & 50 & 34.17 & 14.06 & 23.78 & 1.95 & 1.18 & -12.76 \\
\hline & 60 & 19.23 & -5.72 & 3.40 & 6.73 & 7.92 & -4.09 \\
\hline & 70 & 17.08 & -5.40 & 2.59 & 9.84 & 6.00 & -3.60 \\
\hline & 80 & 21.85 & -4.15 & 4.86 & 1.01 & 2.66 & -8.03 \\
\hline \multirow[t]{3}{*}{50} & 60 & 20.09 & -4.92 & 3.98 & 10.72 & 14.59 & 4.05 \\
\hline & 70 & 14.17 & -7.51 & -2.22 & 12.84 & 8.43 & 0.33 \\
\hline & 80 & 22.08 & -4.91 & 2.84 & 0.83 & 3.34 & -6.73 \\
\hline \multirow[t]{2}{*}{60} & 70 & 13.48 & 6.23 & 16.36 & 14.375 & 3.05 & -2.73 \\
\hline & 80 & 26.82 & -0.65 & 8.91 & -2.82 & -0.83 & -10.61 \\
\hline 70 & 80 & 57.04 & 17.2 & 30.82 & -16.02 & -3.90 & -16.65 \\
\hline
\end{tabular}

with the proposed method. Figure 6 shows CT images of the mouse and the corresponding $Z_{\text {eff }}$ and ED images. Regions of high $Z_{\text {eff }}$ values (bones) are more strongly emphasized in $Z_{\text {eff }}$ images than in other images.

\section{CONCLUSION}

In search of a simple and practical DECT method, we adopted a method originally developed for monochromatic radiation and applied it to DECT of polychromatic x-rays. With errors in a reasonably acceptable range, the proposed DECT method could successfully extract the effective atomic number and electron density information from CT images.

The proposed method showed simplicity of calibration, thus demonstrating its practicality and feasibility. However, further study is needed to improve the accuracy of the extracted information.

\section{ACKNOWLEDGEMENTS}

This work was supported by Nuclear R\&D Program of MEST (Ministry of Education, Science and Technology), Korea, through KOSEF (20110021268)

\section{REFERENCES}

[1] R. E. Alvarez, and A. Macovski, "Energy-selective reconstructions in X-ray computerized tomography," Phys Med Biol, vol. 21, no. 5, pp. 733-44, Sep, 1976.

[2] T. R. Johnson, B. Krauss, M. Sedlmair et al., "Material differentiation by dual energy CT: initial experience," Eur Radiol, vol. 17, no. 6, pp. 1510-7, Jun, 2007.

[3] A. J. Coleman, and M. Sinclair, "A beam-hardening correction using dual-energy computed tomography," Phys Med Biol, vol. 30, no. 11, pp. 1251-6, Nov, 1985.

[4] E. Y. Sidky, Y. Zou, and X. Pan, "Impact of polychromatic $\mathrm{X}$-ray sources on helical, cone-beam computed tomography and dual-energy methods," Phys Med Biol, vol. 49, no. 11, pp. 2293-303, Jun 7, 2004.

[ 5 ] M. J. Guy, "DETECT - Dual energy transmission estimation CT - for improved attenuation correction in SPECT and PET," IEEE Transactions on Nuclear Science, vol. 45, no. 3 PART 2, pp. 1261-1267, 1998.

[6] P. E. Kinahan, A. M. Alessio, and J. A. Fessler, "Dual energy CT attenuation correction methods for quantitative assessment of response to cancer therapy with PET/CT imaging," Technol Cancer Res Treat, vol. 5, no. 4, pp. 319-27, Aug, 2006.

[7] Z. Ying, R. Naidu, and C. R. Crawford, "Dual energy computed tomography for explosive detection," Journal of X-Ray Science and Technology, vol. 14, no. 4, pp. 235256, 2006.

[ 8 ] Z. Ying, R. Naidu, K. Guilbert et al., "Dual energy volumetric X-ray tomographic sensor for luggage screening."

[9] L. A. Lehmann, R. E. Alvarez, A. Macovski et al., "Generalized image combinations in dual KVP digital radiography," Med Phys, vol. 8, no. 5, pp. 659-67, SepOct, 1981.

[10] K. S. Chuang, and H. K. Huang, "A fast dual-energy computational method using isotransmission lines and table lookup," Med Phys, vol. 14, no. 2, pp. 186-92, Mar-Apr, 1987.

[11] G. Zhang, Z. Chen, L. Zhang et al., "Exact Reconstruction for Dual Energy Computed Tomography Using an H-L 
Curve Method." pp. 3485 - 3488.

[12] G. Christ, "Exact treatment of the dual-energy method in CT using polyenergetic X-ray spectra," Phys Med Biol, vol. 29, no. 12, pp. 1501-10, Dec, 1984.

[13] J. C. Steenbeek, C. van Kuijk, J. L. Grashuis et al., "Selection of fat-equivalent materials in postprocessing dual-energy quantitative CT," Med Phys, vol. 19, no. 4, pp. 1051-6, Jul-Aug, 1992.

[14] M. M. Goodsitt, and R. H. Johnson, "Precision in quantitative CT: impact of x-ray dose and matrix size," Med Phys, vol. 19, no. 4, pp. 1025-36, Jul-Aug, 1992.

[15] M. M. Goodsitt, "Beam hardening errors in post-processing dual energy quantitative computed tomography," Med Phys, vol. 22, no. 7, pp. 1039-47, Jul, 1995.

[16] B. J. Heismann, J. Leppert, and K. Stierstorfer, "Density and atomic number measurements with spectral $\mathrm{x}$-ray attenuation method," Journal of Applied Physics, vol. 94, no. 3, pp. 2073-2079, 2003.

[17] P. Sukovic, and N. H. Clinthorne, "Penalized weighted least-squares image reconstruction for dual energy X-ray transmission tomography," IEEE Trans Med Imaging, vol. 19, no. 11, pp. 1075-81, Nov, 2000.

[18] B. De Man, J. Nuyts, P. Dupont et al., "An iterative maximum-likelihood polychromatic algorithm for CT," IEEE Trans Med Imaging, vol. 20, no. 10, pp. 999-1008, Oct, 2001.

[19] J. A. Fessler, I. Elbakri, P. Sukovic et al., "Maximum- likelihood dual-energy tomographic image reconstruction." pp. 38-49.

[20] M. Torikoshi, T. Tsunoo, M. Sasaki et al., "Electron density measurement with dual-energy x-ray CT using synchrotron radiation," Phys Med Biol, vol. 48, no. 5, pp. 673-85, Mar 7, 2003.

[21] M. Torikoshi, T. Tsunoo, Y. Ohno et al., "Features of dual-energy X-ray computed tomography," Nuclear Instruments and Methods in Physics Research, Section A: Accelerators, Spectrometers, Detectors and Associated Equipment, vol. 548, no. 1-2, pp. 99-105, 2005.

[22] C. Rizescu, C. Besöliu, and A. Jipa, "Determination of local density and effective atomic number by the dualenergy computerized tomography method with the 192Ir radioisotope," Nuclear Instruments and Methods in Physics Research, Section A: Accelerators, Spectrometers, Detectors and Associated Equipment, vol. 465, no. 2-3, pp. 584-599, 2001.

[23] H. J. Vinegar, and S. L. Wellington, Method of imaging the atomic number of a sample, US, 1986.

[24] F. Kelcz, P. M. Joseph, and S. K. Hilal, "Noise considerations in dual energy CT scanning," Med Phys, vol. 6, no. 5, pp. 418-25, Sep-Oct, 1979.

[25] G. Poludniowski, G. Landry, F. DeBlois et al., "SpekCalc: a program to calculate photon spectra from tungsten anode x-ray tubes," Phys Med Biol, vol. 54, no. 19, pp. N433-8, Oct 7, 2009. 